\title{
Die Zukunft ist digital - und interprofessionell
}

\section{Fabienne Hohl}

Texterin

Das diesjährige MedEd-Symposium des Schweizerischen Instituts für ärztliche Weiter- und Fortbildung (SIWF) zeigte auf, was die Medizin mit kritischem Knowhow aus der Digitalisierung schöpfen kann und wie wichtig gelebte Interprofessionalität ist. Ferner widmete sich der Anlass berufsethischen Fragen, die sich für die ärztliche Bildung unter Corona noch dringlicher stellen als zuvor.

Die achte Auflage des MedEd-Symposiums verzeichnete über 200 Teilnehmende und war die erste unter der Leitung der neuen SIWF-Präsidentin Monika Brodmann. Past President und Symposiumsgründer Werner Bauer nutzte die Gelegenheit, seiner Nachfolgerin die Leitung des "MedEds» offiziell und «mit gutem Gefühl» zu übergeben. Seit Februar 2021 im Amt, bekundete Brodmann viel Freude an ihrer dynamischen neuen Aufgabe. Als passionierte Bergsteigerin verglich sie das Ziel der kompetenzbasierten Weiterbildung, das Medical Educators heute anstreben, mit dem $\mathrm{K} 2$ in Asien - einem Gipfel, «den man nur im Team ersteigen kann».

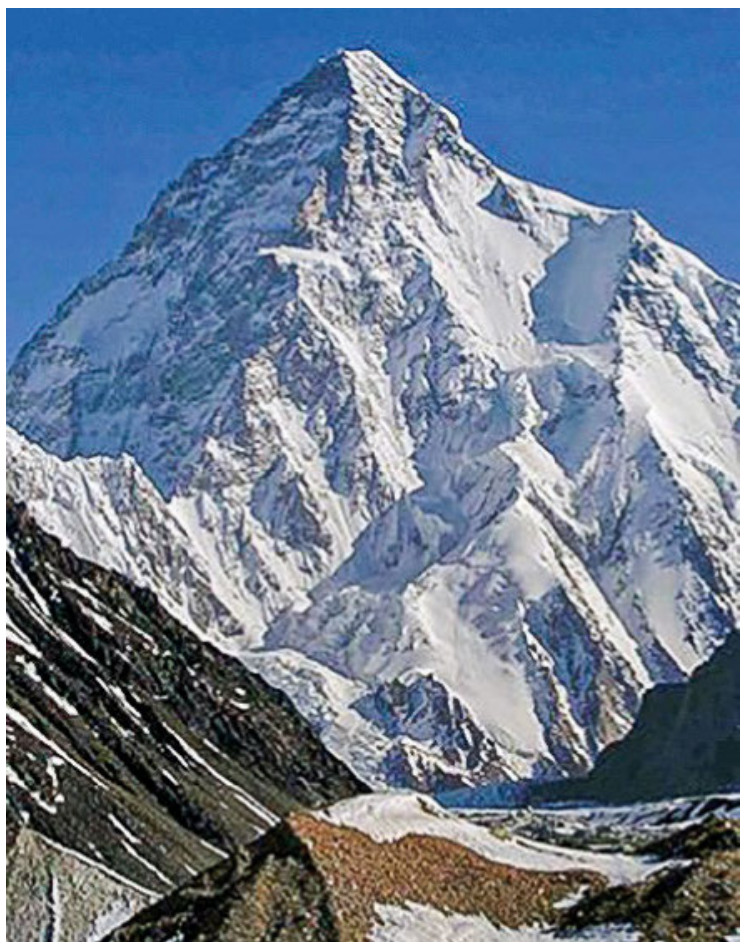

Der Gipfel K2 als Sinnbild für kompetenzbasierte Weiterbildung. Beide sind nur im Team zu erreichen.

\section{Roter Faden Teamwork}

Tatsächlich stand das gesamte Symposium nicht nur thematisch, sondern mit etlichen Co-Referierenden auch organisatorisch auffallend im Zeichen kooperativer Arbeit [1]. Darüber hinaus betonte der Überraschungsgast, FMH-Präsidentin Yvonne Gilli, in ihrem spontanen Grusswort die bewusste und gelebte Kultur des Zusammenwirkens der ärztlichen «Schwesternorganisationen» FMH und SIWF. Zum Thema des Symposiums stellte Gilli fest, dass die Digitalisierung Ärztinnen und Ärzte vor neue Herausforderungen stelle, aber: «Medizin ist immer auch Handwerk und braucht Instrumente. Heute sind dies unter anderem digitale die dem ärztlichen Stand und den Patientinnen und Patienten dienen sollen.» Ein Anliegen, für das sich auch das Bundesamt für Gesundheit (BAG) schon lange gemeinsam mit der Ärzteschaft engagiere, wie der stellvertretende BAG-Direktor Roland Charrière in seiner Ansprache unterstrich. Die Plattform «Zukunft ärztliche Bildung» sei dafür ein geschätzter und bewährter Ort des Austausches.

\section{«Be a good role model»}

Wie über digitale Instrumente verfüge die Medizin auch über ethische Instrumente, eröffnete Samia Hurst, Direktorin des Institut Ethique, Histoire, Humanité an der Universität Genf, die erste Keynote des Tages. «Ethische Prinzipien sind wie eine immaterielle Werkzeugkiste, dank der uns die Patienten vertrauen.» Der Hippokratische Eid beinhaltet diese Prinzipien in klaren Worten - auf den ersten Blick, «but it is a little bit more complicated in practice», kommentierte Hurst. Viele ethische Dilemmata entständen, weil die Ärzteschaft im Berufsleben nicht nur den Patientinnen und Patienten verpflichtet sei, sondern auch den Angehöri- 


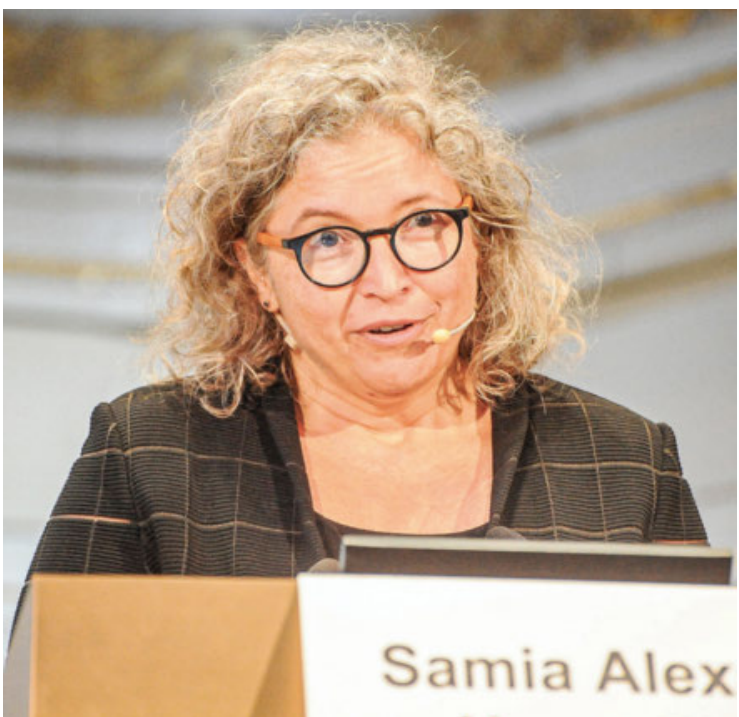

Samia Hurst, Direktorin des Institut Ethique, Histoire, Humanité an der Universität Genf.

gen, den Weiterbildenden, den Vorgesetzten, dem Arbeitgeber, den Behörden, der Öffentlichkeit ... und auch der eigenen Gesundheit. Dilemmata können aber auch entstehen bei Interessenkonflikten der Ärztinnen und Ärzte, die eventuell das Vertrauen der Patientinnen und Patienten gefährden.

Was bedeutet dies nun für die medizinische Bildung? Wohl unterrichte man Ethik in der Humanmedizin, aber ausschlaggebend sei ein qualitativ überzeugendes handwerkliches Training, das auf den Erwerb der dafür notwendigen Kompetenzen fokussiere. «Dies kostet, und darauf müssen wir bestehen», forderte Hurst. Die jungen Ärztinnen und Ärzte müssten konkret lernen, wie man ethische Konflikte erkenne, formuliere und adäquat darauf reagiere. Mit dieser praktischen Vorbereitung gelinge es eher, unprofessionelles Verhalten anzusprechen, sei es das eigene oder jenes von Berufskolleginnen oder -kollegen. Es reiche deshalb nicht, als Institution ethische Guidelines zu publizieren, es brauche eine gelebte Kultur der Ethik. «Wenn sich Theorie und Praxis widersprechen, lernt man, dass die Regeln nicht gelten", stellte Hurst fest, und appellierte an die versammelten Ausbildenden: "You will be a role model - try to be a good one.»

\section{Interprofessionalität macht Schule}

In der nächsten Keynote gaben Thomas Fassier und Patricia Picchiottino, Direktor bzw. stellvertretende Direktorin des Centre interprofessionnel de simulation (CiS) in Genf, Einblick in ihre Arbeit. «Wir müssen Medizin auf allen Ausbildungsstufen interprofessionell vermitteln - nicht nur um die Patientensicherheit zu gewährleisten, sondern auch um die demographischen, klimatischen und epidemiologischen Herausforderungen der Zukunft zu meistern", zeigte sich Fassier überzeugt. Wegbereitend für die interprofessionelle Zusammenarbeit seien die CanMEDS-Rollen gewesen. Diese beschreiben, welche Rollen Ärztinnen und Ärzte in ihrem Beruf einnehmen, und werden in zwischen auch in vielen nicht-ärztlichen Gesundheitsberufen angewandt.

Dabei müsse man sich in einem Team jedoch immer wieder darüber klar werden, was man darunter verstehe, präzisierte Picchiottino. «Nur so lassen sich der gemeinsame Rahmen und die gegenseitige Akzeptanz für die interprofessionelle Zusammenarbeit etablieren, innerhalb der sich alle Fachleute eines Teams äussern können.» Um Interprofessionalität zu lernen, stehen verschiedenste Instrumente zur Verfügung, neben Simulationstraining beispielsweise Rollenspiel, Monitoring oder Shadowing. Fassier unterstrich, dass "Interprofessionalität auch dem medizinischen Nachwuchs aller Gesundheitsberufe ein grosses Bedürfnis ist». Das zeige nicht zuletzt die Gründung der Swiss Youth Health Alliance (SYHA) im Jahr 2019. Die SYHA ist ein von der Swiss Medical Students Association (swimsa) gestartetes Projekt. Sie soll als Bündnis von Studierenden- und Lernenden-Organisationen des Ge-

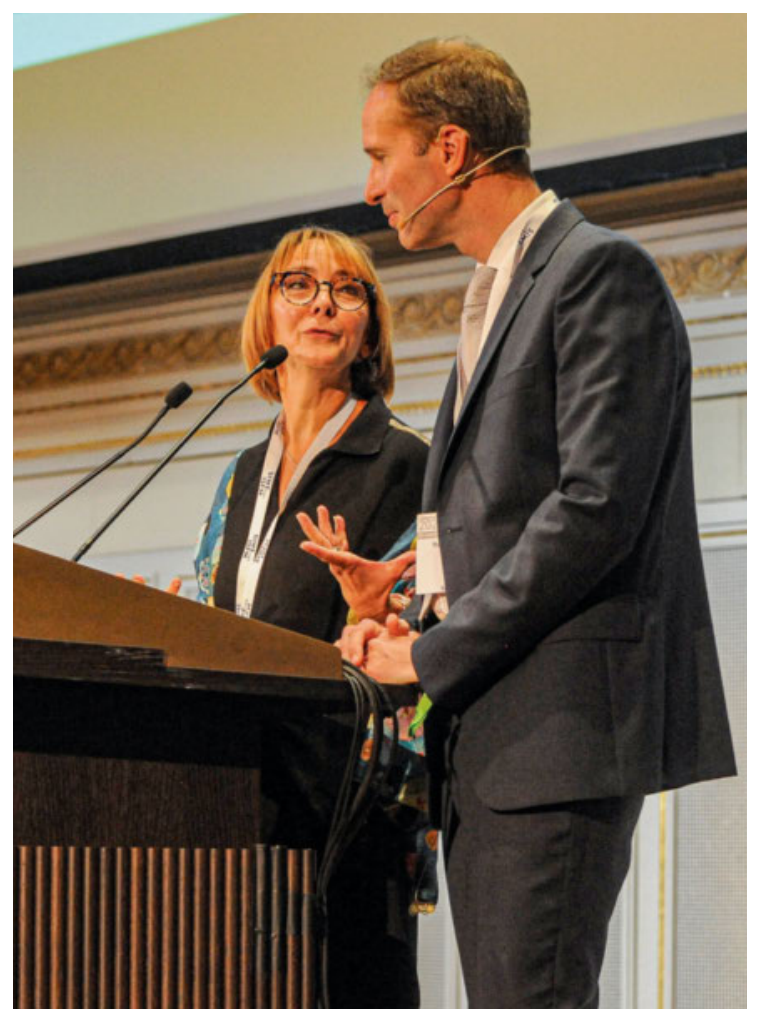

Patricia Picchiottino und Thomas Fassier, stellvertretende Direktorin bzw. Direktor des Centre interprofessionnel de simulation (CiS) in Genf. 
sundheitswesens die Interprofessionalität zwischen den verschiedenen Gesundheitsberufen in der Schweiz fördern. Dessen Ruf nach Weiterbildnerinnen und Weiterbildnern, die Interprofessionalität vorlebten, so Fassier, müsse gehört werden.

\section{Chancen der Digitalisierung}

Dass digitale Kompetenzen an allen Medizinischen Fakultäten zum Einsatz kommen, sind gute Neuigkeiten für Thomas Sauter, Leiter der eNotfallmedizin am Inselspital Bern und Co-Referent der dritten Keynote. Der aktive Einbezug der Studierenden ins Online-Lernen sei zentral, damit sie von der Digitalisierung profitierten, erläuterte er. "Denn die digital natives gehen häufig nicht kompetent um mit virtuellen Inhalten.» Deshalb sei die Online-Lernplattform "enotfallmedizinlearning.ch» nicht als fertiges Lehrwerk konzipiert, sondern als lebendige Lernumgebung mit Grundlagen zum autonomen Wissenserwerb, Möglichkeiten für Feedback und zum Teilen eigener fachlicher Funde. Dabei bewährten sich auch interaktive Tools und Gamification-Elemente wie ein Quizz oder ein Ranking, führte Sauter aus.

Die spielerische Komponente findet sich auch in der simulationsbasierten Ausbildung, welche Co-Referentin

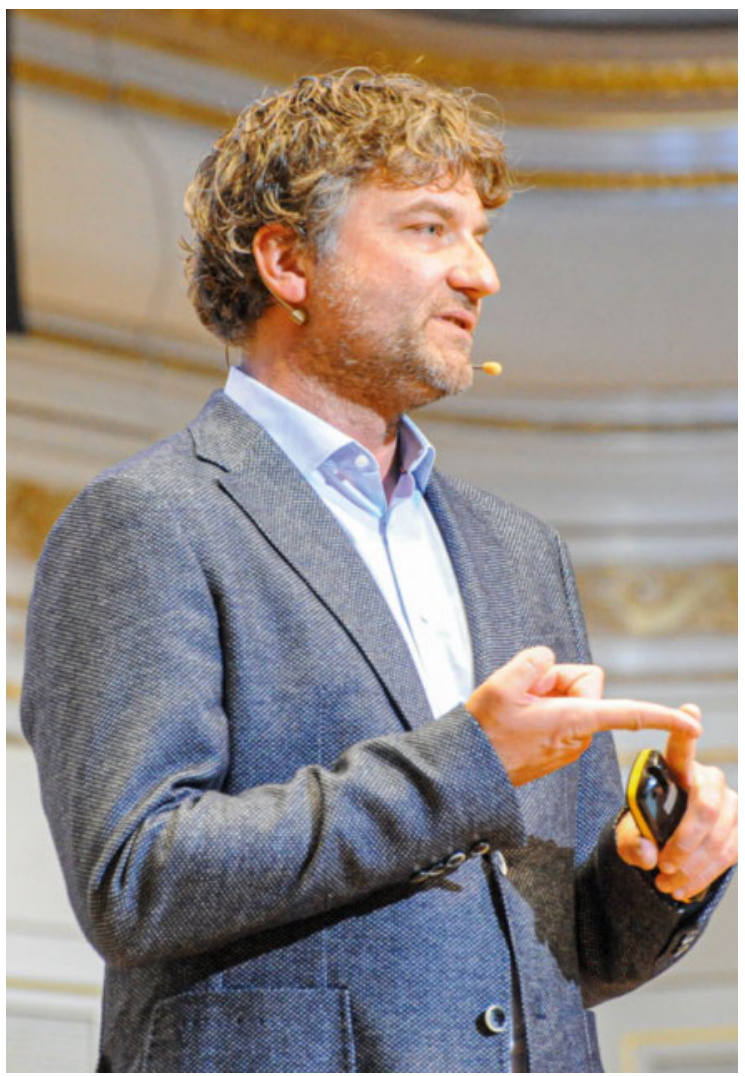

Thomas Sauter, Leiter der eNotfallmedizin am Inselspital Bern.

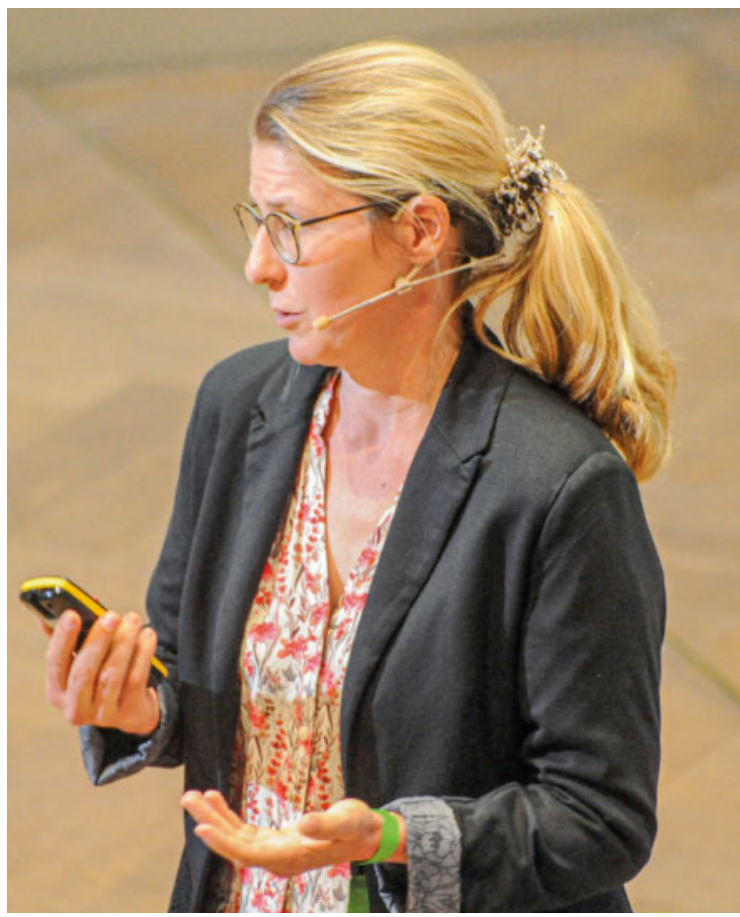

Tanja Birrenbach, Leiterin Arbeitsgruppe Virtual Reality Insel Simulation der digitalen Notfallmedizin am Inselspital Bern.

Tanja Birrenbach vorstellte, ihres Zeichens Leiterin der Arbeitsgruppe Virtual Reality Insel Simulation (ViSL) der digitalen Notfallmedizin am Inselspital. Virtual Reality(VR)-Simulationen eigneten sich bei bestimmten Themen gut für die Medical Education, erklärte Birrenbach. Etwa, um einfache Skills wie das Durchführen eines Covid-Abstrichs oder auch lebensrettende Interventionen zu trainieren, die sich im echten Leben schlecht simulieren lassen oder teuer sind.

Zudem sei VR-Simulationstraining sehr effektiv, wiederholbar, unabhängig von Zeit und Ort, es gebe Feedback und generiere Unmengen von Daten für die Forschung oder für Prüfungen. «Dennoch ist VR keine Wunderwaffe für die Ausbildung der Zukunft", räumte Birrenbach ein. Die Forschungsdesigns seien noch sehr heterogen, die Probandenzahlen klein, und bei den Patientenoutcomes gebe es noch viele Fragen. Trotzdem sind sich Birrenbach und Sauter einig: Die Digitalisierung ist eine Chance, die es in der Medizin und im Teaching mit dem nötigen kritischen Bewusstsein zu nutzen gilt.

\section{Multidisziplinariät als Normalfall}

Nach einer kurzen Mittagspause intensiver Gespräche nahmen die Anwesenden an vier parallel geführten Workshops teil: 
- «Virtuelle Realität in der Bildung» [2]

- «Wie gebe ich gutes klinisches Feedback in der Weiterbildung?» [3]

- «Conflits Interprofessionnels: Expérimentez la Simulation!» [4]

- «Die allgemeinen Lernziele - Stiefkinder der Weiterbildung?» [5]

Anschliessend beleuchtete die vierte Keynote den komplexen Zusammenhang von Körper und Geist. Selma Aybek, Leitende Ärztin für Neuropsychosomatik am Inselspital Bern, brach eine Lanze für ihr Fach das zu Unrecht wenig bekannt sei - als Paradebeispiel für Interprofessionalität. «Die Neuropsychosomatik ist weder schlechte Psychiatrie noch schlechte Medizin. Sie kümmert sich um die Verbindung dieser beiden Bereiche», erklärte sie. So werde, was früher «hysterisch" oder "psychosomatisch" genannt worden sei, heute sachlich als "funktionelle neurologische Störung» bezeichnet. "Dieser neutrale Ausdruck ist gemäss Studien für die Betroffenen zentral», so Aybek. "Damit fühlen sie sich mit ihrem Problem ernst genommen und erhalten nicht einfach eine psychiatrische Diagnose.»

Wohl könnten psychische Faktoren wie übermässiger Stress mit ein Grund für dysfunktionelle Störungen sein, aber es spielten immer auch soziale und genetische Faktoren eine Rolle. Für die Behandlung sei deshalb neben psychiatrischen Massnahmen auch Physiotherapie erfolgversprechend. Aybek wies darauf hin,

\section{SIWF-Award 2021: Die Ausgezeichneten}

Im Rahmen des 8. MedEd-Symposiums wurden auch die Trägerinnen und Träger des SIWF-Award 2021 für besonderes Engagement in der Weiterbildung bekanntgegeben. Diese Auszeichnung erfolgt aufgrund der Nomination durch ehemalige Assistenzärztinnen und -ärzte. Folgende Weiterbildende und Teams erhielten dieses Jahr eine Auszeichnung:

Einzelpersonen: Dr. med. Dr. rer. nat. Steffen Stoewer; Boris Jung, Psychiatrische Klinik Münsterlingen; Prof. Dr. Ekkehard Hewer; Dr. med. Christopher Müssig; Frau Sandra Möhr; Dr. med. Kai Oliver Jensen; Dr. med. MPH Mathias Schlögl; PD Dr. med. Franca Wagner; Dr. med. Pia Bircher; Prof. Dr. med. Jörg Beyer; Dr. med.Yvette Stöckli; Dr. med. Carolie Kretschmer, Monvia Gesundheitszentrum Luzern; Dr. med. Charlotte Seer; Dr. med. Severin Pinilla; Prof. Dr. med. Dr. h.c. Omer Dzemali; Dr. med. Priska Grünig.

Teams: Allgemeine Innere Medizin, Gesundheitszentrum Fricktal, Laufenburg; NZKJ Notfallzentrum für Kinder und Jugendliche, Inselspital Bern; Allgemeine Innere Medizin, Doktorzentrum Wettingen (DZW); Urologie, Kantonsspital Baden; Radio-Onkologie, Kantonsspital St. Gallen; Wirbelsäulenchirurgie und Orthopädie, Schweizer Paraplegiker Zentrum, Nottwil.

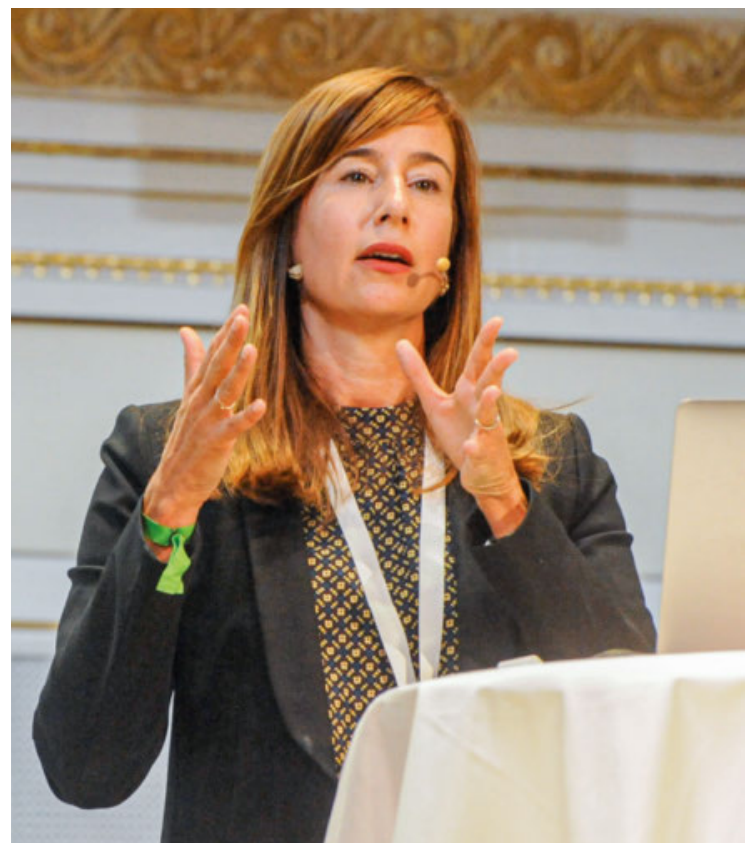

Selma Aybek, Leitende Ärztin für Neuropsychosomatik am Inselspital Bern.

dass die multidisziplinäre Arbeit der Neuropsychosomatik inzwischen internationale Anerkennung erfährt und der European Research Council europaweit $15 \mathrm{PhD}$-Forschungsstellen dafür ausgeschrieben hat. Eine Gelegenheit, die wahrgenommen werden sollte: «Sagen Sie es weiter - wir brauchen Nachwuchs!»

\section{Was wir uns leisten müssen}

Den spannenden Abschluss des MedEd-Symposiums bildete dieses Jahr eine Debatte unter der Leitung von Monika Brodmann. Sie nahm sich gemeinsam mit ihren Gästen Cinzia Zeltner und Elisabeth van Gessel des Themas «Interprofessionalität im Spannungsfeld von Patientensicherung und Ökonomie» an. Schnell wurde klar: Auch wenn Interprofessionalität kurzfristig mehr kostet, das Gesundheitswesen braucht sie dringend und "sie ist schon da", wie Zeltner festhielt. Die BAGVerantwortliche für das Dossier Interprofessionalität führte aus, dass Randregionen mit Versorgungsproblemen das Potenzial des Task Shifting längst erkannt hätten und stark interprofessionell arbeiteten. Dies erhöhe die medizinische Qualität und senke wahrscheinlich langfristig die Kosten.

Ein Votum, das FMH-Vizepräsident Christoph Bosshardt zuspitzte: «Nach den Kosten zu fragen ist müssig - uns gehen die Ärzte aus, bevor das Geld ausgeht. Wir kommen gar nicht darum herum, interprofessionell zu arbeiten.» Ein grosses Potenzial der Interprofessionalität ortete CiS-Gründungsdirektorin van Gessel 


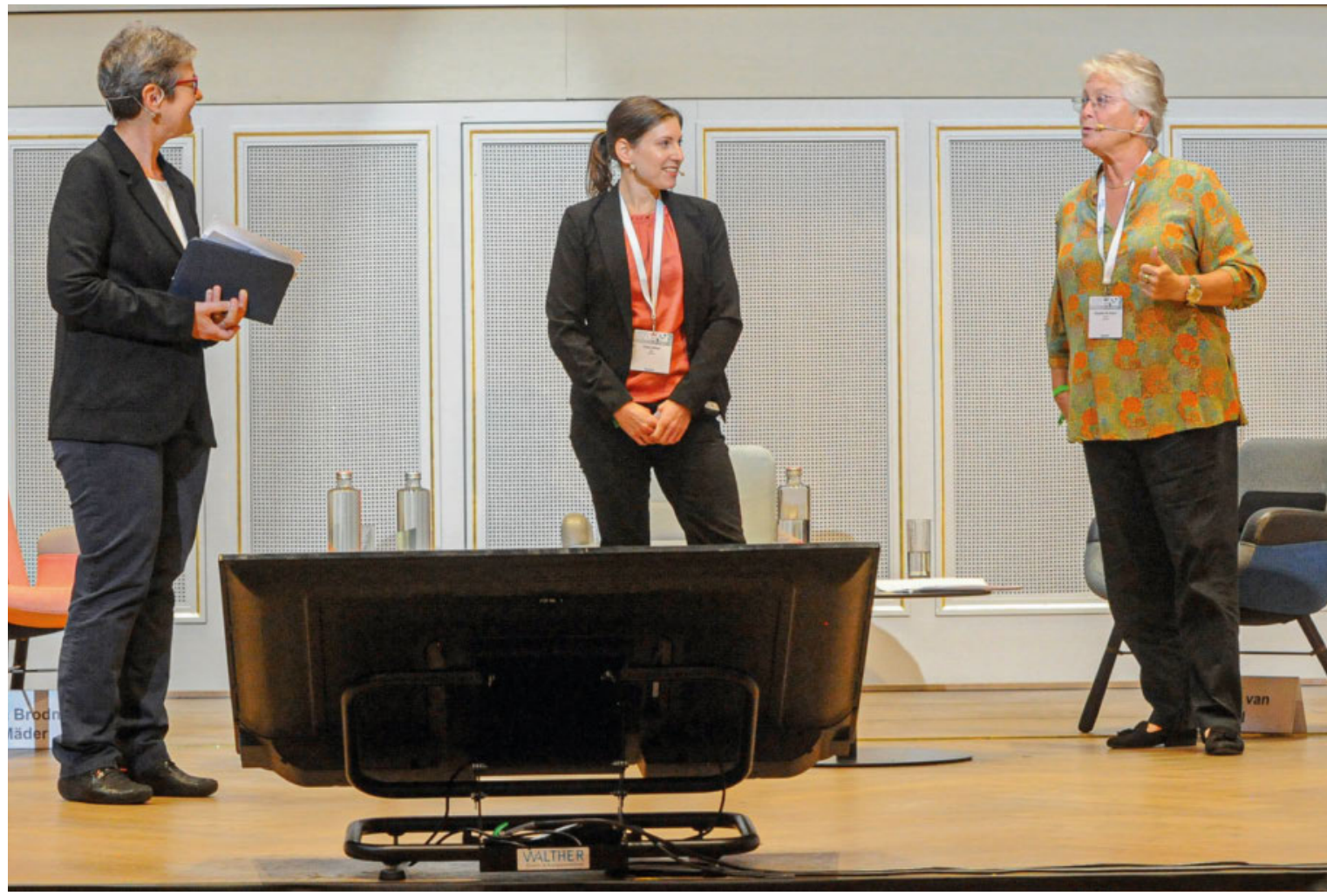

(v.I.n.r.) SIWF-Präsidentin Monika Brodmann im Gespräch mit Cinzia Zeltner vom Bundesamt für Gesundheit (BAG) sowie CiSGründungsdirektorin Elisabeth van Gessel.

im Job Enrichment, das etwa Pflegende dank Task Shifting erführen. «Doch es braucht mehr Innovation», forderte van Gessel, «mehr neue Berufe; es braucht Interprofessionalität, die Patienten und Angehörige einbindet und die vor allem schon in der Ausbildung dazugehört». Eine kraftvolle Vision, die noch den Weg bahnen muss für eine neue Arbeitsrealität, in der Interprofessionalität idealerweise auch gesetzlich verankert ist. Die engagierte Diskussion am MedEd zeigte, dass die ersten Schritte auf diesem Weg bereits getan sind.

Bildnachweis

Medworld AG, ausser Bild auf erster Seite: Monika Brodmann

\section{Literatur}

1 Die Präsentationen zu den Referaten und Workshops sind einsehbar unter: www.siwf.ch/siwf-projekte/meded-symposium.cfm

2 Leitung: Prof. Dr. med. Thomas C. Sauter, MME, Leitender Arzt Universitäres Notfallzentrum, Inselspital, Professor für Telenotfallmedizin, Universität Bern / Dr. med. Tanja Birrenbach, MME,
Oberärztin, Universitäres Notfallzentrum Inselspital Bern, Professur für Telenotfallmedizin, Universität Bern.

3 Leitung: Dr. med. Martin Perrig, MME, Chefarzt, Leiter Bettenstationen, Universitätsklinik für Allgemeine Innere Medizin, Inselspital Bern / Dr. med. Christian Schirlo, MME, Leiter Studienzentrum, Gesundheitswissenschaften und Medizin, Universität Luzern / Dr. Andrea Meienberg, Kaderärztin, Medizinische Poliklinik, Universitätsspital Basel / KD Dr. med. Anke Scheel-Sailer, Leitende Ärztin Paraplegiologie, Physikalischen Medizin und Rehabilitation, Schweizer Paraplegiker-Zentrum, Nottwil.

4 Leitung: Dr méd. Nadia Bajwa, MHPE, Hôpitaux Universitaires de Genève, Université de Genève, Faculté de Médecine / Dr méd. Thomas Fassier, MPH, MHPE, PhD, Directeur du Centre interprofessionnel de Simulation (CiS) / Patricia Picchiottino Zofka, MAS, Directrice adjointe du Centre interprofessionnel de simulation (CiS), Hôpitaux Universitaires de Gèneve (HUG) / Jean-Pierre Bosson, Chargé de formation et de recherche, Centre interprofessionnel de simulation (CiS).

5 Leitung: Dr. med. Werner Bauer, Past President des Schweizerischen Instituts für ärztliche Weiter- und Fortbildung (SIWF), Bern / Dr. med. Patrizia Kündig, Vizepräsidentin, Verband Schweizerischer Assistenz- und Oberärztinnen und -ärzte (VSAO) / Prof. Dr. med. Markus Furrer, Chefarzt und ärztlicher Direktor und Departementsleiter Chirurgie, Kantonsspital Chur / Prof. Dr. Rouven Porz, PhD, Leitung Medizinethik und ärztliche Weiterbildung, Universitätsklinik, Inselspital Bern. 